\title{
Oil spill characterization in the hybrid-polarity SAR domain using log-cumulants
}

\author{
Martine M. Espeseth ${ }^{\mathrm{a}}$, Stine Skrunes ${ }^{\mathrm{a}}$, Camilla Brekke ${ }^{\mathrm{a}}$, Arnt-Børre Salberg ${ }^{\mathrm{b}}$, Cathleen E. \\ Jones $^{\mathrm{c}}$, and Benjamin Holt ${ }^{\mathrm{c}}$ \\ ${ }^{a}$ UiT, The Arctic University of Norway, Hansine Hansens veg 14, 9019 Troms $\varnothing$, Norway \\ ${ }^{\mathrm{b}}$ Norwegian Computing Center, Gaustadalleen 23a, 0373 Oslo, Norway \\ ${ }^{\mathrm{c} J e t}$ Propulsion Laboratory, California Institute of Technology, 4800 Oak Grove Dr., \\ Pasadena, CA, USA 91109
}

\begin{abstract}
Log-cumulants have proven to be an interesting tool for evaluating the statistical properties of potential oil spills in polarimetric Synthetic Aperture Radar (SAR) data within the common horizontal $(\mathrm{H})$ and vertical (V) polarization basis. The use of first, second, and third order sample log-cumulants has shown potential for evaluating the texture and the statistical distributions, as well as discriminating oil from look-alikes. Logcumulants are cumulants derived in the log-domain and can be applied to both single-polarization and multipolarization SAR data. This study is the first to investigate the differences between hybrid-polarity (HP) and full-polarimetric (FP) modes based on the sample log-cumulants of various oil slicks and open water from nine Uninhabited Aerial Vehicle Synthetic Aperture Radar (UAVSAR) scenes acquired off the coast of Norway in 2015 .

The sample log-cumulants calculated from the HP intensities show similar statistical behavior to the FP ones, resulting in a similar interpretation of the sample log-cumulants from HP and FP. Approximately eight hours after release the sample log-cumulants representing emulsion slicks have become more similar to the open water compared to plant oil. We find that the sample log-cumulants of the various oil slicks and open water varies between the scenes and also between the slicks and open water. This might be due to changes in ocean and wind condition, the initial slick properties, and/or the difference in the weathering process of the oil slicks.
\end{abstract}

Keywords: Oil spill characterization, Texture, Log-cumulants, Hybrid-Polarity, SAR, UAVSAR

\section{INTRODUCTION}

Synthetic Aperture Radar (SAR) has been widely used for oil spill monitoring. The SAR instrument is a powerful tool that provides the unique capabilities of penetrating clouds and most weather condition. Depending on the SAR mode used, large spatial coverage, high polarimetric information, and/or fine resolution can be obtained. SAR instruments can be used to detect possible oil spills, and in some cases can be used to characterize the oil spill, i.e., determine properties (dielectric, texture, shape etc.) of the oil that might help discriminate between different types of oil. The measured backscattered intensity in SAR images depends not only on the oil properties, but also on environmental conditions and sensor properties. Oil slicks dampen the waves on the sea surface and occurs as dark pixels in SAR. Therefore a major challenge to using the SAR instruments is distinguishing oil spills from natural phenomena that also dampen the waves and occur as dark pixels at the sea surface. Oil spill detection is considered possible in wind speeds from approximately 1.5 to $15 \mathrm{~m} / \mathrm{s}$, with ideal conditions from 3 - $10 \mathrm{~m} / \mathrm{s}^{1,2}$ The detectability of oil slicks is also considered to be best at incidence angle between $20^{\circ}$ to $45^{\circ} .^{3}$

Single-polarimetric SAR offers large coverage but at the cost of reduced polarimetric information. This mode has been widely used and is the currently used mode in operational oil spill monitoring. In the single channel SAR, texture features have been evaluated as input to classification methods to increase the feature space. Especially, the grey-level co-occurrence matrix (GLCM) has been used (to extract several texture features from the SAR

Further author information: (Send correspondence to Martine M. Espeseth)

E-mail: martine.espeseth@uit.no, Telephone: +47 77623374

Image and Signal Processing for Remote Sensing XXII, edited by Lorenzo Bruzzone,

Francesca Bovolo, Proc. of SPIE Vol. 10004, 1000414 - C) 2016 SPIE

CCC code: $0277-786 \mathrm{X} / 16 / \$ 18 \cdot$ doi: $10.1117 / 12.2241098$

Proc. of SPIE Vol. 10004 1000414-1 
data) (see, e.g., Refs. 4 and 5). More recently, the focus towards full-polarimetric SAR data has intensified, allowing deeper analysis on how various slick types appear in SAR imagery under different environmental and sensor conditions. Polarimetric information, in terms of decomposition and feature extraction, has been widely used in research to analyse oil spills, both for detecting and for discriminating them from look-alikes (see, e.g., Refs. 6-9). The FP mode introduce limitations when it comes to coverage, and the use of HP mode is therefore an important alternative that might allow enough polarimetric information and larger design flexibility than the FP mode. ${ }^{10}$

The compact polarimetry (CP) mode is in the coherent dual-polarimetric (DP) SAR group, where the choice of the transmitted-receive polarizations deviates from the conventional DP SAR. One of the first CP architecture introduced in the SAR literature is the $\pi / 4$ mode, ${ }^{11}$ which transmits a linearly polarized field at $45^{\circ}$, while receiving coherently horizontal and vertical polarizations. Another alternative is to transmit circular polarization and receive coherent dual circular polarizations. ${ }^{12}$ Raney suggested the use of a hybrid variant, by receiving in coherent linear horizontal and vertical while transmitting either left or right circular polarizaton. This alternative was named hybrid-polarity (HP) mode, and has the advantages of a simpler instrument and improved quality of the radar measurements. ${ }^{10}$ The HP mode has already been implemented in the RISAT- 1 and ALOS-2 satellites, and will also be implemented in future SAR missions such as the Canadian RADARSAT Constellation Mission and ISRO. ${ }^{13}$ The potential of the HP mode for oil spill observation has been highlighted by several studies, amongst others in Refs. 14-16. These studies focus on polarimetric features and their ability to observe various oil spills, but do not consider texture features, which will be the focus of this study.

In this study we use the method proposed in 17 (translated in 18) and tested for oil spill observation in 7, 19, and 20. This method evaluates statistical properties of the radar texture, here extracted from several UAVSAR acquisitions, in terms of log-cumulants. Unlike extracting the GLCM, which is more image and pixel focused, the theory of log-cumulants has an underlying assumption of a theoretical model that is based on random variables representing the scattering process at the surface. In the literature, when comparing $\mathrm{CP}$ data to $\mathrm{FP}$ data assumptions are often made. ${ }^{21,22}$ One benefit of using log-cumulants for evaluating the data is that no assumptions - for instance reflection symmetry - are necessary. This study is the first to evaluate log-cumulants for the HP mode.

The main objective of this study is to evaluate whether it is possible to characterize oil spills in the hybridpolarity SAR domain using log-cumulants, in particular: i) to study the texture difference between FP and simulated HP using several acquisitions made using the UAVSAR instrument, ${ }^{23}$ and ii) to study the difference in radar texture of the various oil slicks as they evolve. This paper is organized as follows: Section 2 presents the study area and the data used; the theory of the log-cumulants is presented in Section 3; Section 4 contains the results, and Section 5 presents conclusions from the study.

\section{STUDY AREA AND DATA}

The UAVSAR acquisitions used in this study were collected during a controlled oil spill experiment, the NOrwegian Radar oil Spill Experiment 2015 (NORSE2015), in the North Sea near the Frigg field in June 2015. This experiment was a collaboration between UiT-The Arctic University of Norway, the Jet Propulsion Laboratory (JPL) / National Aeronautics and Space Administration (NASA), and the Norwegian Clean Seas Association for Operating Companies (NOFO). The UAVSAR time series were collected in two flights, approximately three hours between, with 16 and 6 acquisitions in the first and second flights, respectively. The released oils included three emulsions based on the same crude oil, but with varying oil volumetric fraction, i.e., $40 \%$ (E40), 60\% (E60), and $80 \%$ (E80) oil, in addition to one release of plant oil (PO) for simulation of a natural biogenic slick. ${ }^{20,24}$ The UAVSAR monitored the four oil slicks as they evolved and weathered on a high wind driven sea surface (approximately $12 \mathrm{~m} / \mathrm{s}$ ). In addition, X-, C-, and L-band SAR data were also collected from other sensors. The main motivation behind this experiment was to collect multi-sensor and multi-frequency SAR data to study the polarization-dependent electromagnetic signals backscattered from various oil slicks as they evolved on the sea surface. $^{20,24}$ The reader is referred to Refs. 20, 24, and 25 for more information on the experiment.

Table 1 presents details about the released oils, which also are given in 20 and 24 . The four substances were released close in time, left untouched, and imaged from several sensors. 20 and 24 present the two first analyses 
of SAR data collected during this experiment, contain a multi-sensor analysis, and information regarding the in-situ data.

In this study, we use a subset of the UAVSAR scenes that have the same imaging geometry and approximately the same incidence angles where the oil slicks are located. This includes half the time series of 22 images, i.e., nine scenes. The VV-intensity images in decibel $(\mathrm{dB})$ for the investigated scenes are displayed in Fig. 1. These intensity images are geocoded, smoothed, and scaled for displaying purposes. Note that the true width of the images displayed in Fig. 1 varies across the time series as the oil slicks evolve and spread out. The plant oil and the three emulsion slicks are indicated in Fig. 1, with the PO as the southern-most slick, followed by E40, E60, and E80. The release of the E80 oil was not complete in the first acquisition. Hence, this scene is left out in the analysis when evaluating the E80 slick. The properties of the UAVSAR sensor are given in Table 2. One of the benefits of the UAVSAR instruments is the low noise floor. The noise equivalent sigma zero (NESZ), is in the range -48 to $-33 \mathrm{~dB}^{23}$ for the UAVSAR, which is low compared to other sensors like Radarsat-2 (NESZ in the range -27.5 to $-43 \mathrm{~dB}^{26}$ ) and TerraSAR-X (NESZ in the range $-19 \mathrm{~dB}$ to $-26 \mathrm{~dB}^{27}$ ). A consequence of returns below the NESZ is corrupted signals, and challenges occur when attempting to extract information from these signals. The UAVSAR scenes used in this analysis have values that are above the noise floor for both the open water regions and the various oil slicks at the given incidence angle, even for the cross-polarization (cross-pol) channel. The cross-pol channel is not used in this analysis, due to space limitation. Therefore, the texture analysis presented in this study is performed on more or less noise free data.

\section{BACKGROUND}

This section covers the background theory of the HP mode and the log-cumulants.

\subsection{HP Theory}

The fundamental quantities measured by a polarimetric SAR system are the complex backscattering components $\mathrm{S}_{i j}$. Here, $i$ and $j$ define the polarizations of the transmitting and the receiving polarimetric channels in the radar system. For the FP mode, the scattering vector in the lexicographic base is defined as

$$
k_{F P}=\left[S_{h h}, S_{h v}, S_{v h}, S_{v v}\right]^{T},
$$

where $\mathrm{T}$ denotes the transpose operator, and $h$ and $v$ denote horizontal and vertical polarizations. For the HP mode using the right $(\mathrm{r})$ circular transmit the HP scattering vector is defined as

$$
k_{H P}=\left[S_{r h}, S_{r v}\right]^{T} .
$$

In the linear horizontal and vertical basis the HP scattering vector is expressed as ${ }^{21}$

$$
k_{H P}=\frac{1}{\sqrt{2}}\left[S_{h h}-i S_{h v},-i S_{v v}+S_{v h}\right]^{T} .
$$

The UAVASAR instrument offers full-polarimetric capabilities with high resolution and high signal-to-noise ratio. Real HP data was not available for this study, hence the HP scattering components are simulated based on Eq. (3).

\subsection{Product Model}

In this study we use multi-look intensity (MLI) data, which is created by:

$$
I_{(x y)}=\frac{1}{L} \sum_{j=1}^{L}\left|S_{j,(x y)}\right|^{2}
$$

where $\mathrm{x}$ and $\mathrm{y}$ denote the polarization at the transmitter and receiver, and $\mathrm{L}$ is the number of samples included in the computation of the multi-look intensities. In this study the intensity data have been multi-looked with 3 looks in range and 12 looks in azimuth direction (same as in Ref. 6). The MLI is further smoothed with a $5 \times$ 
5 pixel sliding window to reduce speckle. The log-cumulants described in the next section are calculated based on the smoothed MLI data.

In the product model, the backscattered signal is expressed as the product of two separate processes, namely speckle and texture. From the central limit theorem, the distribution of fully developed speckle for the real and imaginary parts of the complex scattering vector is Gaussian. This article will focus on the multi-look intensities (MLIs) extracted from the UAVSAR data. To allow us to describe and quantify both the effect of speckle and texture we start with the known product model (see, e.g., 28 and 29) for the MLI case, i.e.:

$$
I=T S,
$$

where $I$ is the MLI, $T$ represents the texture, and $S$ represents the speckle. For textureless areas (homogeneous) and for fully developed speckle the MLI is Gamma distributed, ${ }^{30}$ and Gaussian statistics are in place. This indicates that the fluctuations in the radar cross-section is only due to speckle. ${ }^{30}$ Our goal is to evaluate the presence of texture for the various oil slicks using both the FP and HP polarimetric mode, and observe how the texture changes as a function of time.

\subsection{Mellin Kind Statistics}

A given distribution can be described by its statistical moments and cumulants. In classical statistics, the Fourier transform is used in the characteristic function and in the cumulant generating function when generating moments and cumulants, respectively. Instead of using the Fourier transform, the author 17 (translated in 18) proposed to use the Mellin kind transform, which lead to the Mellin kind characteristic function and Mellin kind moments and cumulants, also named log-moments and log-cumulants. The reader is referred to 17 and 18 for additional information on the theory of the Mellin kind statistics.

The log-cumulants are used as a measure of the deviation from Gaussian statistics, in cases where other distributions are present. In this study, the one dimensional (with respect to polarimetric channels) MLIs are considered, i.e., the univariate case in the Mellin kind statistics is used. The first order log-cumulant $\left(\kappa_{1}\right)$ is the $\log$ of the backscattering intensity (MLI). The second- and third order log-cumulants, i.e., $\kappa_{2}$ and $\kappa_{3}$, represent variance and skewness in the log-domain, and are used to evaluate both the texture and speckle within the data. $^{31}$ These are calculated as; ${ }^{17}$

$$
\begin{gathered}
\kappa_{1}=m_{1} \\
\kappa_{2}=m_{2}-m_{1}^{2} \\
\kappa_{3}=m_{3}-3 m_{1} m_{2}+2 m_{1}^{3}
\end{gathered}
$$

where $m_{i}$ is the log-moment of order $i$ of a sample of $\mathrm{N}$ intensities, ${ }^{17}$ i.e.,:

$$
m_{i}=\frac{1}{N} \sum_{n=1}^{N}\left(\log I_{n}\right)^{i} .
$$

This study calculates $\kappa_{1}, \kappa_{2}$, and $\kappa_{3}$ based on the MLIs from the VV and HH channels. These are then compared to the $\kappa_{1}, \kappa_{2}$, and $\kappa_{3}$ generated from the simulated RV and RH MLIs. Following the product model in Eq. (5), the log-cumulant is a sum of the log-cumulant of the texture and the speckle, i.e.. ${ }^{17,18}$

$$
\kappa_{\nu}=\kappa_{\nu}^{(T)}+\kappa_{\nu}^{(S)}
$$

where $\nu$ represents the order of the log-cumulant. In this study, we are interested in analysing the texture component, hence the $\kappa_{\nu}^{(S)}$ is subtracted from the $\kappa_{\nu}$. The area of interest in this analysis is affected by high wind $(\sim 12 \mathrm{~m} / \mathrm{s})$, and from the analysis in the upcoming section, it is clear that texture is also present in the open water regions. Hence, we therefore subtract the speckle component based on a theoretical estimate of the texture. Since fully developed speckle is assumed to follow a Gamma distribution for MLIs we use this distribution when estimating the speckle component of the log-cumulants. The authors in 17 and 18 used the first $\psi(1, \cdot)$ and 
second $\psi(2, \cdot)$ order polygamma function as estimates for the second and third order log-cumulants. The new second and third order log-cumulants are updated as follow: ${ }^{17,18}$

$$
\begin{aligned}
& \tilde{\kappa}_{2}=\kappa_{2}-\psi(1, L) \\
& \tilde{\kappa}_{3}=\kappa_{3}-\psi(2, L)
\end{aligned}
$$

where $L$ is the number of looks, which is related to the shape parameter for the speckle distribution. In the following section the second and third order sample log-cumulants, calculated from the MLIs, are compared to the second and third order log-cumulants from various model distributions.

\section{RESULTS AND DISCUSSION}

This section presents illustrations and discussions on the sample log-cumulants calculated from the data. This includes log-cumulant diagrams, and a discussion of how the texture varies with time and the overall difference between the sample log-cumulants between FP and simulated HP.

\subsection{Log-cumulant diagram}

The oil slicks are segmented by using the "extended polarimetric feature space" (EPFS), an unsupervised segmentation method. ${ }^{32,33}$ This method includes both polarimetric and textural information from the SAR data, and groups all pixels with similar statistical properties in the same clusters using the expectation maximisation (EM) algorithm, assuming a multivariate Gaussian model for the input data. Additionally, a discrete Markov random field (MRF) contextual smoothing stage completes the segmentation by integrating contextual information to improve the connectivity among the image segments. The reader is referred to 32 and 33 for additional information on this segmentation approach. The oil slicks are manually selected and labeled based on the in-situ data after the segmentation. For each scene, regions of open water are selected at the same location as the oil slicks to limit the incidence angle effect. The sample log-cumulants are calculated from a selection of 4000 data points from the areas representing plant oil, open water and the three emulsion slicks (E40, E60 or E80). The selection is performed using Bootstrap sampling, and is repeated 800 times for each region of interest (ROI).

Figs. 2 to 5 show the log-cumulant diagrams calculated from the VV, HH, RV, and RH intensity, respectively. The log-cumulant diagrams include the span of some well-known texture models in the $\kappa_{2}-\kappa_{3}$ space. More specifically for MLI case, the Gamma, inverse Gamma, Beta, inverse Beta, and Fisher distributions. The intensities are one dimensional, hence the univariate case is considered. More information on these model distributions and the interpretation of the diagram can be found in 17 and 18. The cross-pol component is left out in this analysis. From the diagrams (in Figs. 2 to 5) we can evaluate the estimated distribution of the texture for the various regions, i.e., the open water and the oil slicks. This can be performed by studying the location of the sample log-cumulants of the various regions relative to the theoretical distributions indicated in these diagrams.

Figs. 2 and 4 show that the sample log-cumulants from the VV intensity exhibit a similar trend to those derived from the RV intensity. This observation is based on the location of the clusters through the time series. The same observation holds for the sample log-cumulants from the $\mathrm{HH}$ and $\mathrm{RH}$ intensity in Figs. 3 and 5 . In Figs. 2 and 4, the open water regions lie closer to the theoretical delta distribution compared to the oil slicks regions. This indicates less texture compared to the oil slick regions. The $\tilde{\kappa}_{2}$ is related to the internal variation within the sample that it is calculated from. The E60 slick in both Figs. 2 and 4 shows larger deviation from Gaussian statistics (in the Fisher distribution region) in the first two acquisitions compared to the other slicks. In the third acquisition, the E80 and E60 slicks have similar deviation from Gaussian statistics, but the E80 slick is closer to the theoretical Gamma distribution, while the E60 slicks is closer to the theoretical inverse Gamma distribution. Following the fourth acquisition to the seventh acquisition, the E80 is the slick that has the largest deviation from the Gaussian statistics. This might be a result of the higher oil content of the E80 slick compared to the other slick types. The E40 slick has the highest water fraction, and was the emulsion slick that was released first. The E40 slick's log-cumulants are located between the Fisher and inverse Gamma distribution in the first five acquisitions, and approaches the same values as the open water regions from the sixth acquisition onward (in both Figs. 2 and 4). In the same figures, the PO's values show a different trend than the values of 
the emulsion slicks. The plant oil is close to the theoretical delta distribution (Gaussian statistics) in the first acquisition, and a larger deviation from Gaussian statistics is observed in the following acquisitions. In the last two acquisitions, the plant oil is the region that has the largest deviation from Gaussian statistics, indicating a higher texture for this region. Hence, the plant oil seems to "hold on" to it's texture longer compared to the emulsion slicks in Figs. 2 and 4.

In Figs. 3 and 5, the E60 slick shows the largest deviation from Gaussian statistics in the first four acquisitions, while the log-cumulants for the E80 slick shows the largest deviation from Gaussian statistics from the fifth to the eight acquisition. The E40 slick, from the $\mathrm{HH}$ and RH case, shows a similar trend to the VV and RV case. In the majority of the acquisitions, the plant oil cluster is located in the inverse Gamma and inverse Beta distribution regions. Skrunes et al. ${ }^{7,19}$ found that the texture for plant oil was less than for the emulsion slick using both Cand X-band SAR. This conclusion agrees with some of the results we find here for the UAVSAR scenes in the early stages of the oil slicks.

A parallel study of the UAVSAR time series investigated the oil slicks drift (using an oil drift model) (see 25). The model results indicated that the PO entrained more quickly and deeper into the water column compared to the emulsion slicks. Additionally, the PO droplets resurfaced to maintain the observable slick. As a result of the entrainment into the water column, PO is shielding from the wind drag and Stokes drift, and later resurfacing resulted in longer visibility on the sea surface. The same study also compared the E80 slick with the model simulations, and the model results showed that the simulated E80 had $\sim 50 \%$ or more of the oil on the surface throughout the simulation, and relatively little penetrated deeper than 10 meters. Hence, the emulsion slicks were more exposed to wind drag and Stokes drift, which resulted in more spreading particularly along the wind direction and possibly less visibility in the SAR images than the PO. This trend might be reflected in the log-cumulant since the log-cumulants for the plant oil seems to have a different trend than the emulsion slicks.

The relative arrangement between the various clusters is not consistent across the time series in the $\log$ cumulant diagrams, which limits the possibility to discriminate the various oil slicks. It is possible to separate the slicks of different oil content using the diagrams but not classify them according to the type of oil in the slick based upon the log-cumulants alone. This might be due to several factor concerning the data used, like the high wind and that the oils were small in volume when they were released. From Fig. 1 we can observe a strong wave pattern across the intensity images. This also affects the statistical properties, which contributes to internal variation within the sample that is used when calculating the log-cumulants. ${ }^{20}$ The challenge remains in further work to understand how the log-cumulants change with the oil weathering process as well as the ocean and wind state.

\subsection{Texture as a function of time}

The sample log-cumulants of the open water regions change slightly across the time series using VV, RV, HH, and RH intensities. This could be due to several factors, primarily the change in the ocean and wind state, which can affect the texture of the surface. The open water regions are selected approximately at the same incidence angle range along the time series, so changes in texture due to incidence angle is limited. The authors in 19 subtracted the log-cumulants representing the open water from the log-cumulants calculated from the various oil slick regions. This was done to compare the log-cumulant across several SAR scenes. As a result of this procedure the difference in the texture between oil slick and open water is left in the modified log-cumulants. The same procedure is performed in this study, to enable a comparison in the radar texture along the acquisitions. Hence, in this part of the analysis we normalize the sample log-cumulants, i.e.,:

$$
\begin{aligned}
& \tilde{\tilde{\kappa}}_{1}=\kappa_{1}-\left\langle\kappa_{1}^{(O W)}\right\rangle \\
& \tilde{\kappa}_{2}=\kappa_{2}-\left\langle\kappa_{2}^{(O W)}\right\rangle \\
& \tilde{\kappa}_{3}=\kappa_{3}-\left\langle\kappa_{3}^{(O W)}\right\rangle
\end{aligned}
$$

where $\tilde{\tilde{\kappa}}_{1}, \tilde{\tilde{\kappa}}_{2}$, and $\tilde{\tilde{\kappa}}_{3}$ are now representing the difference in texture (log-cumulants) between the oil slicks and the open water regions. Note, in the previous section we subtracted a theoretical estimate of the speckle component, while in this case we normalize the sample log-cumulants based on the log-cumulants from the open water sample. 
Figs. 6 and 7 show the $\tilde{\tilde{\kappa}}_{1}, \tilde{\tilde{\kappa}}_{2}$, and $\tilde{\tilde{\kappa}}_{3}$ as function of time for the VV and HH, respectively. In the previous section we observed a high similarity between VV and RV, and HH and RH. Hence, the $\tilde{\tilde{\kappa}}_{1}, \tilde{\tilde{\kappa}}_{2}$, and $\tilde{\tilde{\kappa}}_{3}$ as function of time for the RV and RH case is left out due to space limitation. The upper panel in Figs. 6 and 7 display the mean $\tilde{\tilde{\kappa}}_{1}$ as a function of time for the various ROIs. The center and lower panels show $\tilde{\tilde{\kappa}}_{2}$ and $\tilde{\tilde{\kappa}}_{3}$ as function of time, respectively. The thicknesses of the graphs showing $\tilde{\tilde{\kappa}}_{2}$ and $\tilde{\tilde{\kappa}}_{3}$ indicate the mean \pm standard deviation of the given log-cumulant.

In Fig. 6 the VV $\tilde{\tilde{\kappa}}_{1}$ representing the open water regions is above the VV $\tilde{\tilde{\kappa}}_{1}$ for the various oil slicks. This indicates that the backscattered response is higher for the open water regions. In the first acquisition, the E40 and E60 have the lowest backscattered response using the VV and HH intensity. Following the second acquisition to the end of the time series, the plant oil has the lowest $\tilde{\tilde{\kappa}}_{1}$ for all the intensities. A higher difference between the plant oil $\tilde{\tilde{\kappa}}_{1}$ and the $\tilde{\tilde{\kappa}}_{1}$ for the emulsion slicks is observed for the HH intensity compared to the VV intensity. Both $\tilde{\tilde{\kappa}}_{2}$ and $\tilde{\tilde{\kappa}}_{3}$ from the HH intensity show similar trends to the VV intensity. The $\tilde{\tilde{\kappa}}_{2}$ representing the PO increases with time, while the $\tilde{\tilde{\kappa}}_{2}$ for E40 and E60 decreases with time. For the E80 slick, the first acquisition is left out since this oil was not released yet, and the $\tilde{\tilde{\kappa}}_{2}$ for the E80 slick increases with time, and then decreases in the last part of the time series. The same $\tilde{\tilde{\kappa}}_{2}$ trend for the emulsion slicks is observed for the HH intensity. The time series for PO for the HH intensity, however, shows a slightly different trend than in the VV intensity. The $\tilde{\tilde{\kappa}}_{2}$ for the PO fluctuates around the $\tilde{\tilde{\kappa}}_{2}$ for the OW in the HH case. The $\tilde{\tilde{\kappa}}_{3}$ representing the plant oil shows first positive values of $\tilde{\tilde{\kappa}}_{3}$ and negative values in the last two acquisitions. $\tilde{\kappa}_{3}$ for E40 slicks decreases towards zero, indicating that the difference between $\kappa_{3}$ for the open water and $\kappa_{3}$ for the E40 slick is small. $\tilde{\tilde{\kappa}}_{3}$ for E60 slick increases in the first part of the time series and then decreases in the last part in the VV case. $\tilde{\tilde{\kappa}}_{3}$ for E80 slicks shows a more fluctuating trend, but with an overall increase towards zero, where the texture difference between E80 and OW is small. A difference trend of $\tilde{\tilde{\kappa}}_{3}$ for the E60 and E80 slicks are observed for the HH case. In this case, $\tilde{\tilde{\kappa}}_{3}$ for the E60 slick decreases between the first three acquisitions, and then decreases in the last seven acquisitions. The $\tilde{\tilde{\kappa}}_{3}$ for E80 is on the positive side of $\tilde{\tilde{\kappa}}_{3}$ for the open water, which was the opposite as the $\tilde{\tilde{\kappa}}_{3}$ for the VV case. Overall, the VV and HH $\tilde{\tilde{\kappa}}_{2}$ (representing the internal variations) from the three emulsion slicks decreases in the end of the time series. This trend is not seen for the plant oil, where the behavior is a bit different between HH and VV $\tilde{\tilde{\kappa}}_{2}$. Here, the VV $\tilde{\tilde{\kappa}}_{2}$ increases in the end of the time series, while the HH $\tilde{\tilde{\kappa}}_{2}$ fluctuates along the time series, indicating a clear sensitivity between the two polarization channels for the plant oil.

\subsection{Texture difference between FP and HP}

The results presented in sections 4.1 and 4.2 show that the sample log-cumulants calculated from the RV intensity are similar to the ones calculated from the VV intensities, and the same was observed for the HH versus $\mathrm{RH}$ intensities. In the following, a direct comparison between FP and HP sample log-cumulants are presented through scatter-plots. A pairwise comparison between the three log-cumulants from FP and HP representing the various oils and open water region are shown in Figs. 8 and 9. The different colors represent the UAVSAR acquisitions, and the sample log-cumulants from FP is plotted on the x-axis, while the sample log-cumulants from the simulated HP intensity is plotted along the y-axis. Note that the scale on the $\mathrm{x}$ - and $\mathrm{y}$-axes varies between the panels. If the sample log-cumulants from FP and simulated HP intensity are similar, the marker should lie along the diagonal line. The Pearson correlation coefficient between FP and simulated HP is also calculated for each pair of the sample log-cumulants.

High correlation between $\kappa_{1}$ from $\mathrm{VV}(\mathrm{HH})$ and $\kappa_{1}$ from $\mathrm{RV}(\mathrm{RH})$ intensity is observed along the time series for the various oil slicks and the open water region. The Pearson correlation coefficient gave high values (above 0.98 ) in all cases. The scatterplots are shifted towards the right for the various $\kappa_{1}$, indicating higher values of FP $\kappa_{1}$ compared to HP $\kappa_{1}$. This shift is a result of the power difference of $-3 \mathrm{~dB}$ when simulating HP from the FP data (see Eq. (3)). ${ }^{11}$

The correlation between $\kappa_{2}$ from VV and RV is also high (as seen in the second column of Fig. 8), and most of the marker lies along the diagonal line, indicating high similarity between $\kappa_{2}$ from VV and RV intensities. This is also the case for HH versus RH, as seen in the second column of Fig. 9. The diagonal line lies at the center of the clusters of points for the various oil slicks for $\kappa_{2}$ for both the VV versus RV and HH versus RH 
intensities. For the open water region, however, the clusters are shifted more to the right, indicating higher texture for the FP compared to the HP case.

The difference between $\kappa_{3}$ from the VV and RV intensities are shown in the third column in Fig. 8. Here, the clusters are more circular, indicating higher variance in the difference between the two $\kappa_{3}$. This is also the case for the $\mathrm{HH}$ versus RH in Fig. 9. Again, the diagonal lines lie through the center of these clusters, indicating low bias in the difference between the two $\kappa_{3}$. Based on visual inspections of Figs. 8 and 9 we can conclude that the correlation between the log-cumulants from the FP and HP intensities are high.

\section{CONCLUSION}

A comparison between FP and simulated HP data using sample log-cumulants from a UAVSAR time series has been presented. The study is based on nine UAVSAR scenes with approximately equal incidence angle at the oil slick locations. The scenes contain four different slicks, i.e., plant oil and three emulsion slicks, and are collected under high wind conditions.

High similarity and correlation between the log-cumulants from FP and HP intensities were also found, yielding equal interpretation of the radar texture from either of these modes. Given the benefit of the HP mode, i.e., more flexibility in terms of calibration, power, and mass,${ }^{10}$ the HP mode could be a better alternative than the FP mode for practical implementation. The log-cumulants as a function of time have different behavior depending on the slick type. These observations could be due to several factors like change in ocean and wind state or their variations between the locations of the different slicks, the initial slick properties, and the different weathering processes of the various slicks. This was more pronounced when using the log-cumulants generated from the VV and RV intensities compared to the ones generated from the $\mathrm{HH}$ and $\mathrm{RH}$ intensities. Approximately eight hours after release the sample log-cumulants representing the emulsion slicks have become more similar to the open water compared to the plant oil. The VV and HH $\tilde{\tilde{\kappa}}_{2}$ (representing the internal variations) from the three emulsion slicks decreases in the end of the time series. This trend is not seen for the plant oil, where the behavior is a bit different between HH and VV $\tilde{\tilde{\kappa}}_{2}$. Here, the VV $\tilde{\tilde{\kappa}}_{2}$ increases in the end of the time series, while the $\mathrm{HH} \tilde{\tilde{\kappa}}_{2}$ fluctuates along the time line, indicating a clear difference between the two polarization channels $(\mathrm{HH}$ and VV) for the plant oil.

The sample log-cumulant diagrams show that the relative arrangement between the various clusters is not consistent through the time series. The high temporal variability in the slicks' texture limits the use of these parameters in discrimination of the various oil slicks, but it is still possible to separate them using the diagrams, however a clear rule of classification cannot be obtained.

Further work to be done includes the use of the whole UAVSAR time series of 22 scenes because only the scenes with the same imaging geometry are used in this study. In this analysis we generated the log-cumulant from the multi-looked intensities, but it is also possible to generate them from the sample covariance matrix, which should be tested on both the FP and simulated (or real) HP covariance matrix. Also, the potential use of log-cumulants as a texture measure for real HP data should also be investigated, which could be done on RISAT-1 data that actually offer the HP mode with right-circular transmitted pulses. The conclusions drawn in this analysis are based on one time series, and further investigation on several time seres of evolving oil slicks with various properties and under different wind conditions should be conducted.

\section{ACKNOWLEDGMENTS}

The authors would like to thank NOFO for hosting NORSE2015 and MET Norway for collecting the met/ocean observations. This research was carried out in part at the Jet Propulsion Laboratory, California Institute of Technology, under contract with the National Aeronautics and Space Administration. NORSE2015 was partly financed by CIRFA (RCN Grant no. 237906). Thanks also to Anthony Doulgeris at UiT for discussions on both the theory of log-cumulants and the segmentation method. UAVSAR data are courtesy of NASA/JPL Caltech. 


\section{REFERENCES}

[1] Solberg, A. H. S., "Remote Sensing of Ocean Oil-Spill Pollution," Proceedings of the IEEE, 100(10), 29312945 (2012).

[2] Fingas, M. and Brown, C., "Review of oil spill remote sensing," Marine Pollution Bulletin, 83(1), 9 - 23 (2014).

[3] Girard-Ardhuin, F., Mercier, G., Collard, F., and Garello, R., "Operational oil-slick characterization by SAR imagery and synergistic data," IEEE Journal of Oceanic Engineering 30(2), 487-495 (2005).

[4] Xue, X., Wang, X., Xiang, F., and Wang, H., "A New Method of SAR Image Segmentation Based on the Gray Level Co-Occurrence Matrix and Fuzzy Neural Network," 2010 6th International Conference on Wireless Communications Networking and Mobile Computing (WiCOM) , 1-4 (2010).

[5] Wei, L., Hu, Z., Guo, M., Jiang, M., and Zhang, S., "Texture feature analysis in oil spill monitoring by SAR image," 2012 20th International Conference on Geoinformatics , 1-6 (2012).

[6] Minchew, B., Jones, C. E., and Holt, B., "Polarimetric Analysis of Backscatter From the Deepwater Horizon Oil Spill Using L-Band Synthetic Aperture Radar," IEEE Transaction on Geoscience and Remote Sensing 50(10), 3812-3830 (2012).

[7] Skrunes, S., Brekke, C., Eltoft, T., and Kudryavtsev, V., "Comparing Near-Coincident C- and X-Band SAR Acquisitions of Marine Oil Spills," IEEE Transaction on Geoscience and Remote Sensing 53(4), 1958-1975 (2015).

[8] Skrunes, S., Brekke, C., and Eltoft, T., "Characterization of Marine Surface Slicks by Radarsat-2 Multipolarization Features," IEEE Transactions on Geoscience and Remote Sensing 52(9), 5302-5319 (2014).

[9] Migliaccio, M., Gambardella, A., and Tranfaglia, M., "SAR polarimetry to observe oil spills," IEEE Transaction on Geoscience and Remote Sensing 45(2), 506-511 (2007).

[10] Raney, R. K., "Hybrid-Polarity SAR Architecture," IEEE Transactions on Geoscience and Remote Sensing 45(11), 3397-3404 (2007).

[11] Souyris, J.-C. and Mingot, S., "Polarimetry based on one transmitting and two receiving polarizations: the $\pi / 4$ mode," Geoscience and Remote Sensing Symposium, 2002. IGARSS '02. 2002 IEEE International 1, 629-631 (24 - 28 June 2002).

[12] Stacy, N. and Preiss, M., "Compact polarimetric analysis of X-band SAR data," In EuSAR 2006: Proceedings of the 6th European Conference on Synthetic Aperture Radar, Dresden, Germany. VDE Verlag GMBH, Frankfurt, Germany (16 - 18 May 2006).

[13] Raney, R. K., "A Perspective on Compact Polarimetry," IEEE Geoscience and Remote Sensing Newsletters 160, 12-18 (2011).

[14] Salberg, A.-B., Rudjord, O., and Solberg, A. H. S., "Oil Spill Detection in Hybrid-Polarimetric SAR Images," IEEE Transactions on Geoscience and Remote Sensing 52(10), 6521-6533 (2014).

[15] Shirvany, R., Chabert, M., and Tourneret, J.-Y., "Ship and Oil-Spill Detection using the Degree of Polarization in Linear and Hybrid/Compact Dual-Pol SAR," IEEE Journal of Selected Topics in Applied Earth Observations and Remote Sensing 5(3), 885 - 892 (2012).

[16] Collins, M. J., Denbina, M., Minchew, B., Jones, C. E., and Holt, B., "On the Use of Simulated Airborn Compact Polarimetric SAR for Characterizing Oil-Water Mixing of the Deepwater Horizon Oil Spill," IEEE Journal of Selected Topics in Applied Earth Observations and Remote Sensing 8(3), 1062-1077 (2015).

[17] Nicolas, J.-M., "Introduction aux Statistiques de deuxième espèce: applications des Logs-moments et des Logs-cumulants à l'analysis des lois d'images radar," Traitement du Signal 19(3), 139-167 (2002).

[18] Nicolas, J.-M. and Anfinsen, S. N., "Introduction to second kind statistics: Application of log-moments and log-cumulants to the analysis of radar image distributions," Technical note translation from French of Ref. 16 (2012).

[19] Skrunes, S., Brekke, C., and Doulgeris, A. P., "Characterization of SAR Low Backscatter Ocean Features Using Log-Cumulants," IEEE Geoscience and Remote Sensing Letters 12(4), 836 - 840 (2015).

[20] Skrunes, S., Brekke, C., Jones, C. E., and Holt, B., "A Multisensor Comparison of Experimental Oil Spills in Polarimetric SAR for High Wind Conditions," IEEE Journal of Selected Topics in Applied Earth Observations and Remote Sensing (2016). 
[21] Nord, M. E., Ainsworth, T. L., Lee, J.-S., and Stacy, N., "Comparison of Compact Polarimetric Synthetic Aperture Radar Modes," IEEE Transactions on Geoscience and Remote Sensing 47(1), 634-646 (2009).

[22] Souyris, J.-C., Imboa, P., Fjørtoft, R., Mingot, S., and Lee, J.-S., "Compact Polarimetry Based on Symmetry Properties of Geophysical Media: The $\pi / 4$ Mode," IEEE Transactions on Geoscience and Remote Sensing 43(3), 634-646 (2005).

[23] Fore, A. G., Chapman, B. D., Hawkins, B. P., Hensley, S., Jones, C. E., Michel, T. R., and Muellerschoen, R. J., "UAVSAR Polarimetric Calibration," IEEE Transactions on Geoscience and Remote Sensing 53(6), 3481-3491 (2015).

[24] Brekke, C., Jones, C., Skrunes, S., Holt, B., Espeseth, M., and Eltoft, T., "Cross-Correlation Between Polarization Channels in SAR Imagery Over Oceanographic Features," IEEE Geoscience and Remote Sensing Letters 13(7), 997-1001 (2016).

[25] Jones, C. E., Dagestad, K.-F., Breivik, Ø., Holt, B., Röhrs, J., Christensen, K. H., Espeseth, M., Brekke, C., and Skrunes, S., "Measurement and Modeling of Oil Slick Transport," Journal of Geophysical Research: Oceans (2016 (in review)).

[26] Slade, B., "Radarsat-2 product description," MacDonald, Dettwiler and Associates Ltd. (2011).

[27] Fritz, T. and Eineder, M., "TerraSAR-X ground segment basic product specification document," DLR (2010).

[28] Oliver, C. and Quegan, S., [Understanding Synthetic Aperture Radar Images], 2nd ed. Raleigh, USA: SciTech Publishing Inc. (2004).

[29] Touzi, R., Boerner, W. M., Lee, J.-S., and Lüneburg, E., "A review of polarimetry in the context of synthetic aperture radar: Concepts and information extraction," Canadian Journal of Remote Sensing 30(3), 380-407 (2004).

[30] Bruniquel, J. and Lopès, A., "On the True Multilook Intensity Distribution in SAR Imagery," in Proceedings IEEE International Geoscience and Remote Sensing Symposium Proceedings 1(1-3) (1998).

[31] Anfinsen, S. N. and Eltoft, T., "Application of the matrix-variate mellin transform to analysis of polarimetric radar images," IEEE Transaction on Geoscience and Remote Sensing 49(6), 2281-2295 (2011).

[32] Doulgeris, A. P. and Eltoft, T., "Scale mixture of Gaussian modelling of polarimetric SAR data," EURASIP Journal on Advances in Signal Processing (8) (2010).

[33] Doulgeris, A. P. and Eltoft, T., "An advanced non-Gaussian feature space method for Pol-SAR image segmentation," In Proceedings of IEEE International Geosicence and Remote Sensing Symposium 5(22-26) (2013).

Table 1. Properties of the experimental oil releases during NORSE2015. ${ }^{20,24}$

\begin{tabular}{|c|c|c|c|}
\hline Release & Time (UTC) & Substance & Volume \\
\hline $\mathrm{PO}$ & $04: 48$ & Plant Oil: Radiagreen ebo & $0.2 \mathrm{~m}^{3}$ \\
\hline E40 & $04: 59$ & Emulsion ( $40 \%$ oil) & $0.5 \mathrm{~m}^{3}$ \\
\hline E60 & $05: 15$ & $\begin{array}{l}300 \mathrm{~L} \text { water }+100 \mathrm{~L} \text { Troll }+100 \mathrm{~L} \text { Oseberg }+0.2 \mathrm{~L} \text { One-Mul } \\
\text { Emulsion }(60 \% \text { oil }) \\
200 \mathrm{~L} \text { water }+150 \mathrm{~L} \text { Troll }+100 \mathrm{~L} \text { Oseberg }+0.2 \mathrm{~L} \text { One-Mul }\end{array}$ & $0.5 \mathrm{~m}^{3}$ \\
\hline E80 & $05: 30$ & $\begin{array}{l}\text { Emulsion }(80 \% \text { oil }) \\
100 \mathrm{~L} \text { water }+200 \mathrm{~L} \text { Troll }+100 \mathrm{~L} \text { Oseberg }+0.2 \mathrm{~L} \text { One-Mul }\end{array}$ & $0.5 \mathrm{~m}^{3}$ \\
\hline
\end{tabular}


Table 2. Properties of the UAVSAR sensor ${ }^{23}$ and the acquired time series.

\begin{tabular}{|l|l|l|l|l|l|l|l|l|}
\hline $\begin{array}{l}\text { Time }^{a} \\
(\mathrm{UTC})\end{array}$ & Mode & Polarization & $\begin{array}{l}\text { Frequency } \\
(\mathrm{GHz})\end{array}$ & $\begin{array}{l}\text { Incidence } \\
\text { angle } \\
(\mathrm{deg})\end{array}$ & $\begin{array}{l}\text { NESZ } \\
(\mathrm{dB})\end{array}$ & $\begin{array}{l}\text { Resolution } \\
\left(\mathrm{rg}^{b} \times \mathrm{az}^{c}\right) \\
(\mathrm{m})\end{array}$ & $\begin{array}{l}\text { Swath } \\
\text { width } \\
(\mathrm{km})\end{array}$ & $\begin{array}{l}\text { Look } \\
\text { direction }\end{array}$ \\
\hline $\begin{array}{l}05: 32- \\
13: 18\end{array}$ & PolSAR & $\begin{array}{l}\text { Full-pol } \\
\text { (hh,hv, } \\
\text { vh,vv })\end{array}$ & $\begin{array}{l}\text { L-band } \\
(1.26)\end{array}$ & 19.5 to 67.5 & $\sim-48$ to -33 & $2.5 \times 0.9$ & 20 & Left \\
\hline
\end{tabular}

${ }^{a}$ From start to end of acquisition, ${ }^{b} \mathrm{rg}$ : range, ${ }^{c}$ az: azimuth 

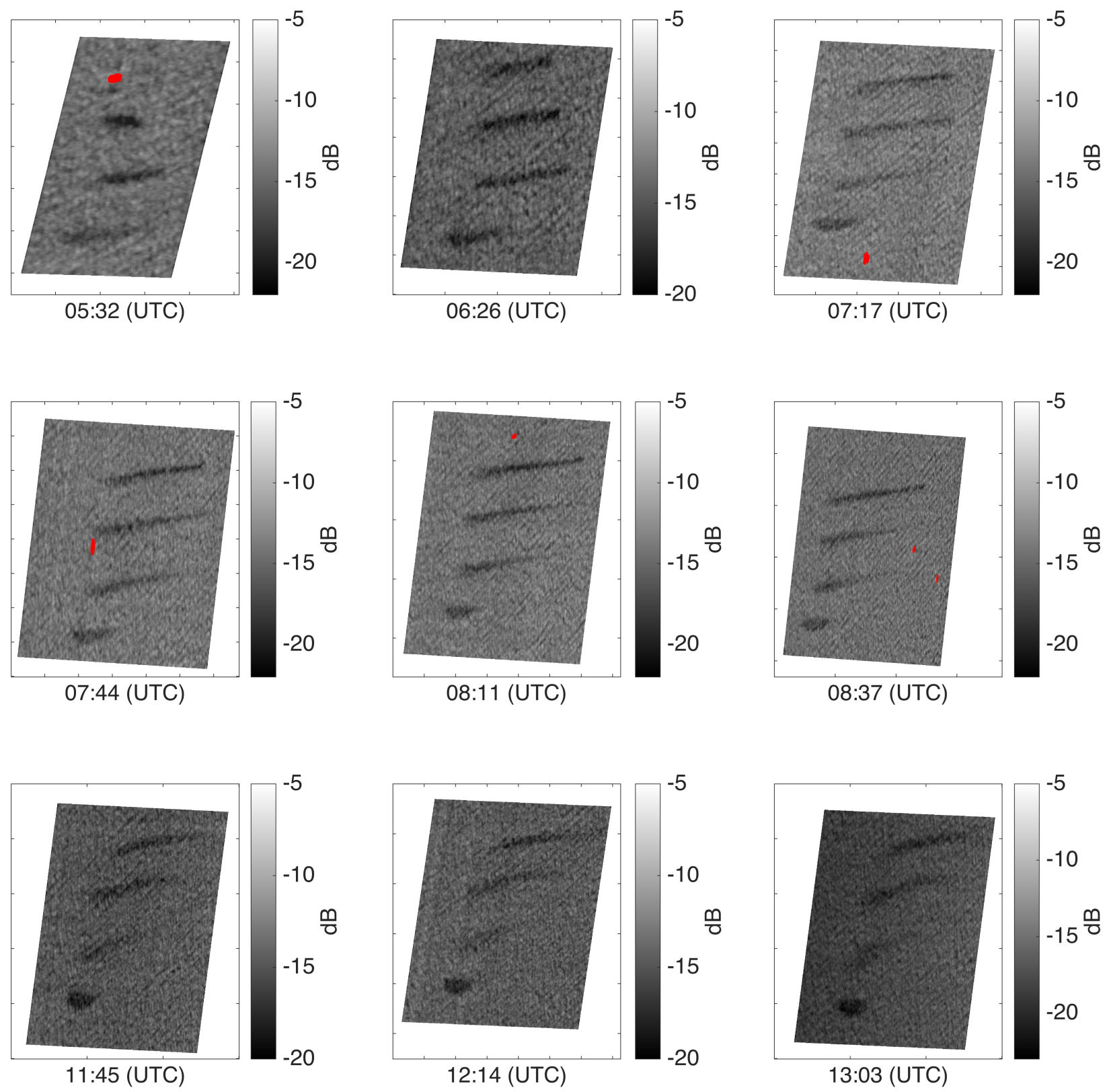

Figure 1. Geocoded VV-intensity images in $\mathrm{dB}$ for the ascending UAVSAR scenes. The ships are masked out and colored red. UAVSAR data is courtesy of NASA/JPL-Caltech. 

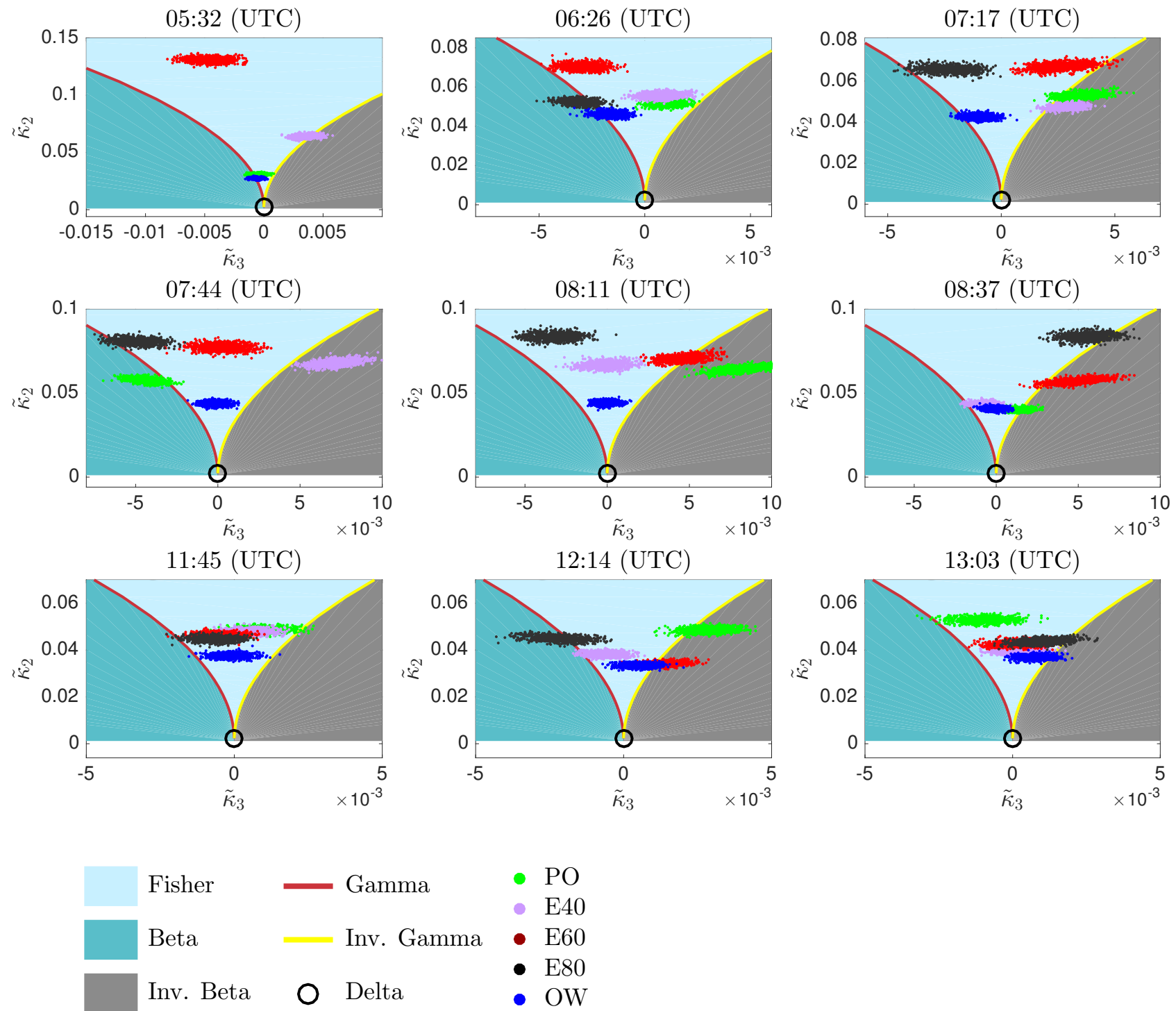

Figure 2. $\tilde{\kappa}_{2}$ and $\tilde{\kappa}_{3}$ from the multi-looked VV intensity plotted in the log-cumulant diagram. Theoretical model distributions are also indicated in these diagrams. 

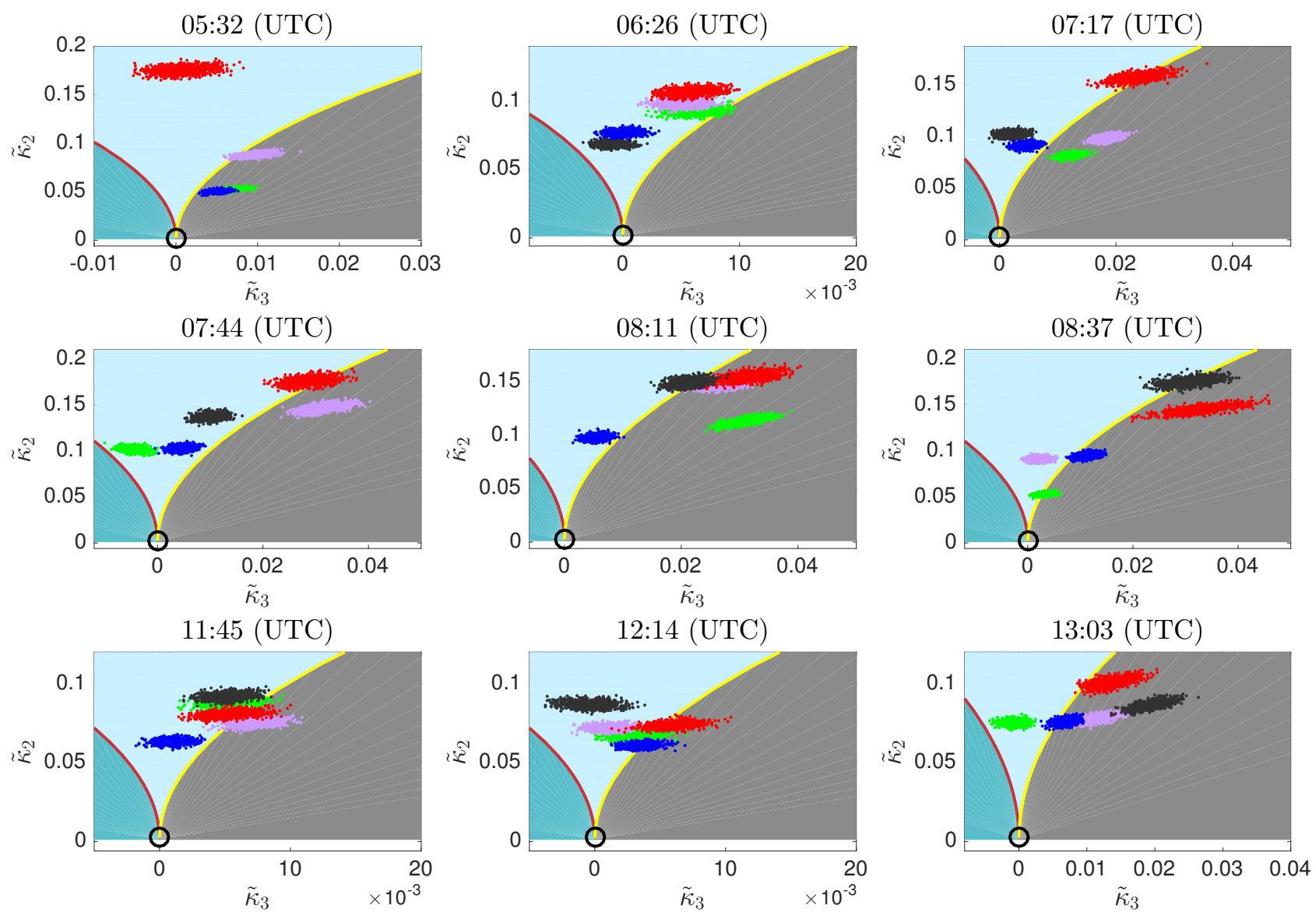

$\begin{array}{llll}\text { Fisher } & & \text { Gamma } & \bullet \mathrm{PO} \\ \text { Beta } & & \text { Inv. Gamma } & \bullet \mathrm{E} 60 \\ \text { Inv. Beta } & \mathrm{O} \text { Delta } & \bullet \mathrm{E} 80 \\ & & \bullet \mathrm{OW}\end{array}$

Figure 3. $\tilde{\kappa}_{2}$ and $\tilde{\kappa}_{3}$ from the multi-looked HH intensity plotted in the log-cumulant diagram. Theoretical model distributions are also indicated in these diagrams. 

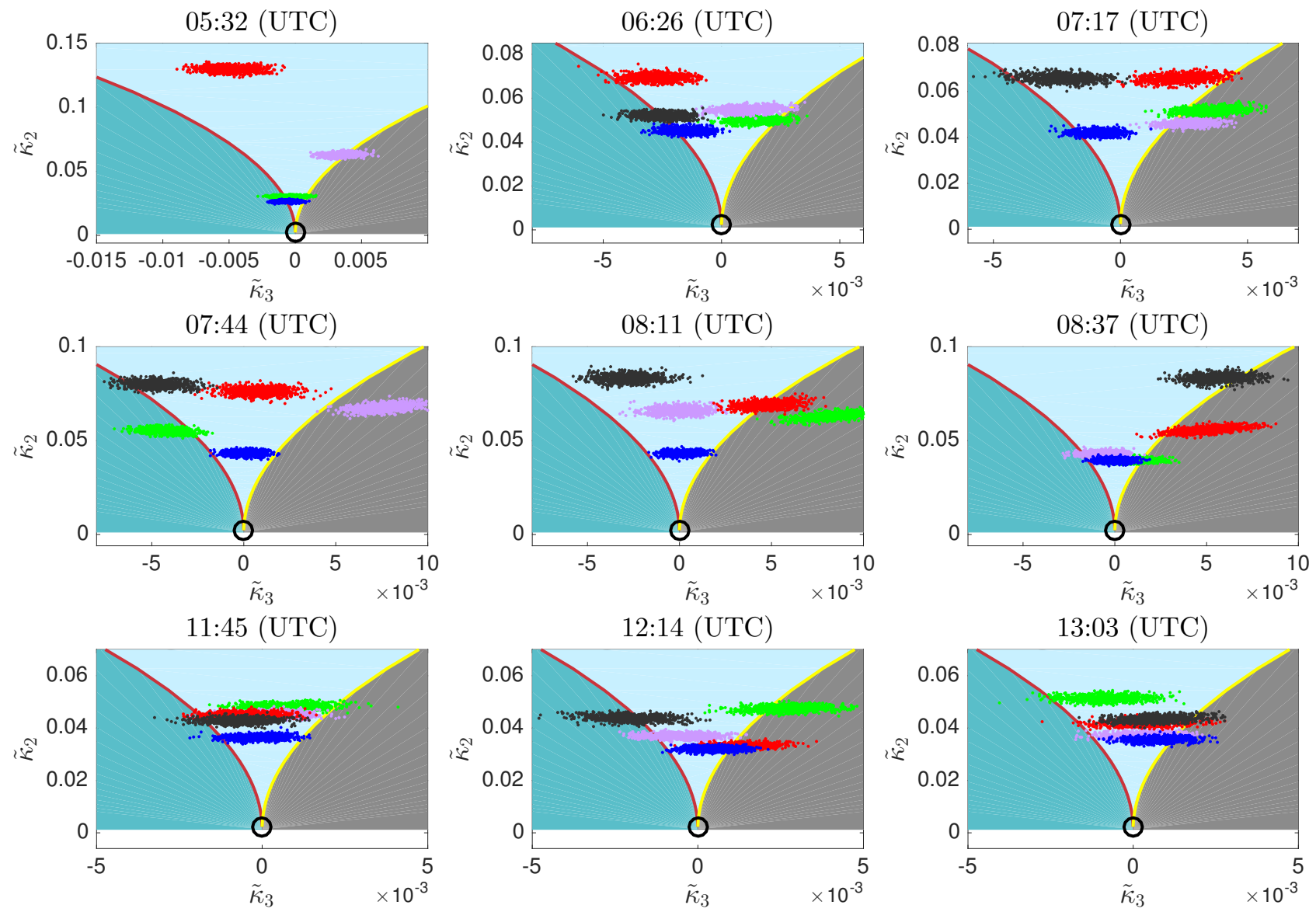

\begin{tabular}{|c|c|c|}
\hline Fisher & 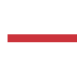 & Gamma \\
\hline Beta & 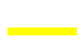 & Inv. Gamma \\
\hline Inv. Beta & 0 & Delta \\
\hline
\end{tabular}

Figure 4. $\tilde{\kappa}_{2}$ and $\tilde{\kappa}_{3}$ from the multi-looked RV intensity plotted in the log-cumulant diagram. Theoretical model distributions are also indicated in these diagrams. 

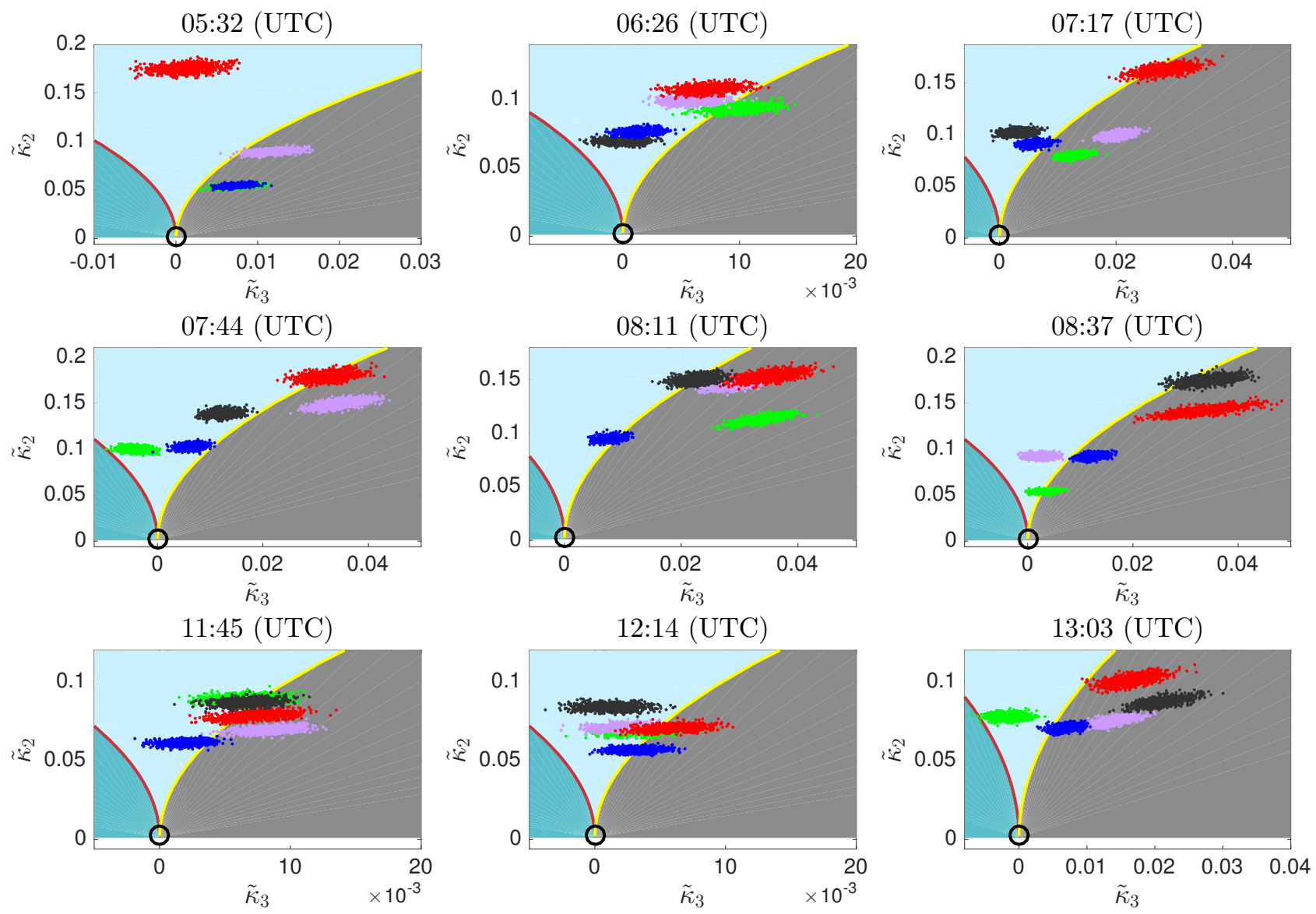

$\begin{array}{llll}\text { Fisher } & & \text { Gamma } & \bullet \mathrm{PO} \\ \text { Beta } & & \text { Inv. Gamma } & \bullet \mathrm{E} 60 \\ \text { Inv. Beta } & \mathrm{O} \text { Delta } & \bullet \mathrm{E} 80 \\ & & \bullet \mathrm{OW}\end{array}$

Figure 5. $\tilde{\kappa}_{2}$ and $\tilde{\kappa}_{3}$ from the multi-looked RH intensity plotted in the log-cumulant diagram. Theoretical model distributions are also indicated in these diagrams. 

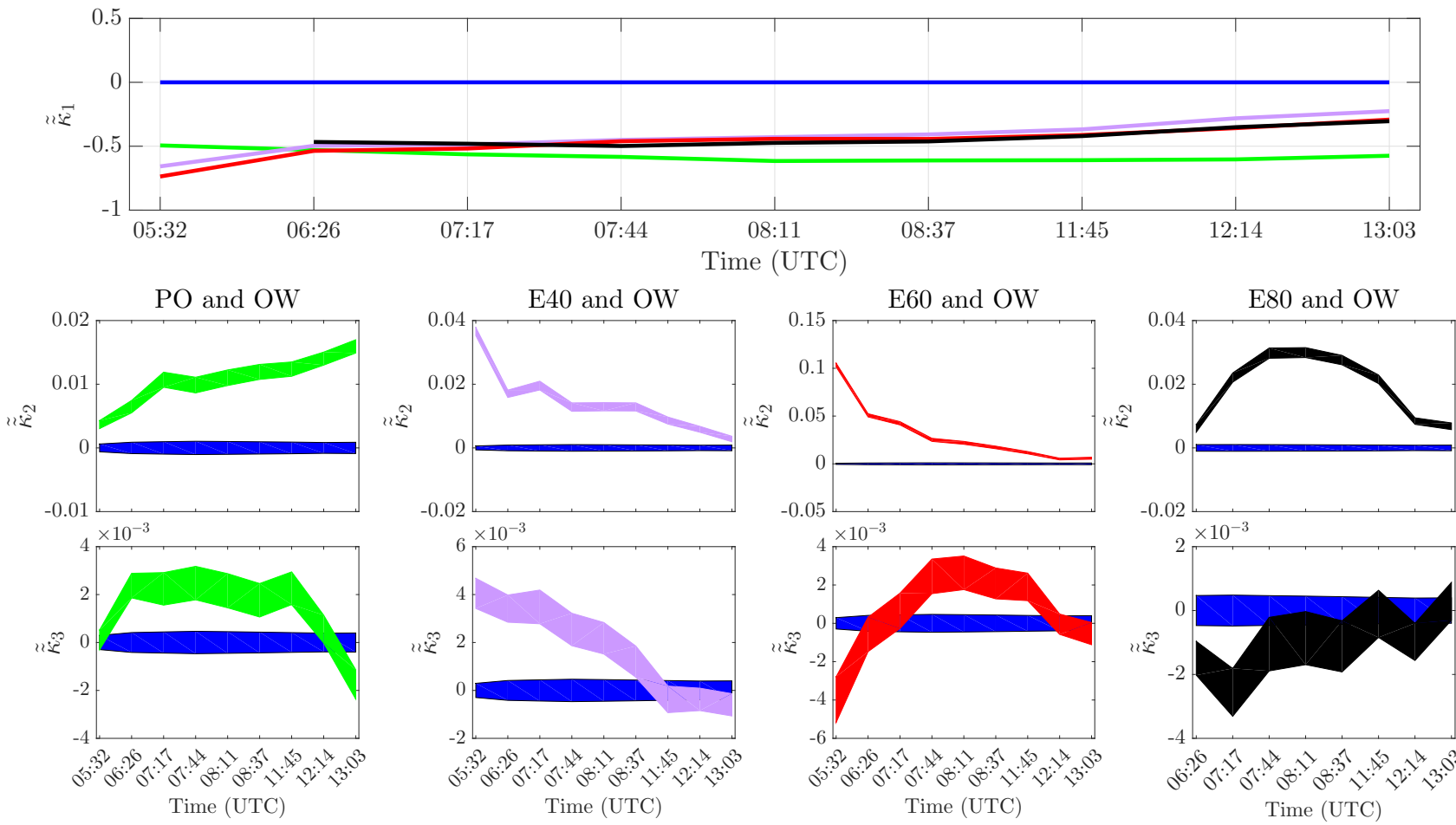

Figure 6. $\tilde{\tilde{\kappa}}_{1}, \tilde{\tilde{\kappa}}_{2}$ and $\tilde{\tilde{\kappa}}_{3}$ from the multi-looked VV intensity as a function of time. The thickness of $\tilde{\tilde{\kappa}}_{2}$ and $\tilde{\tilde{\kappa}}_{3}$ plots represents the variance within the log-cumulants.
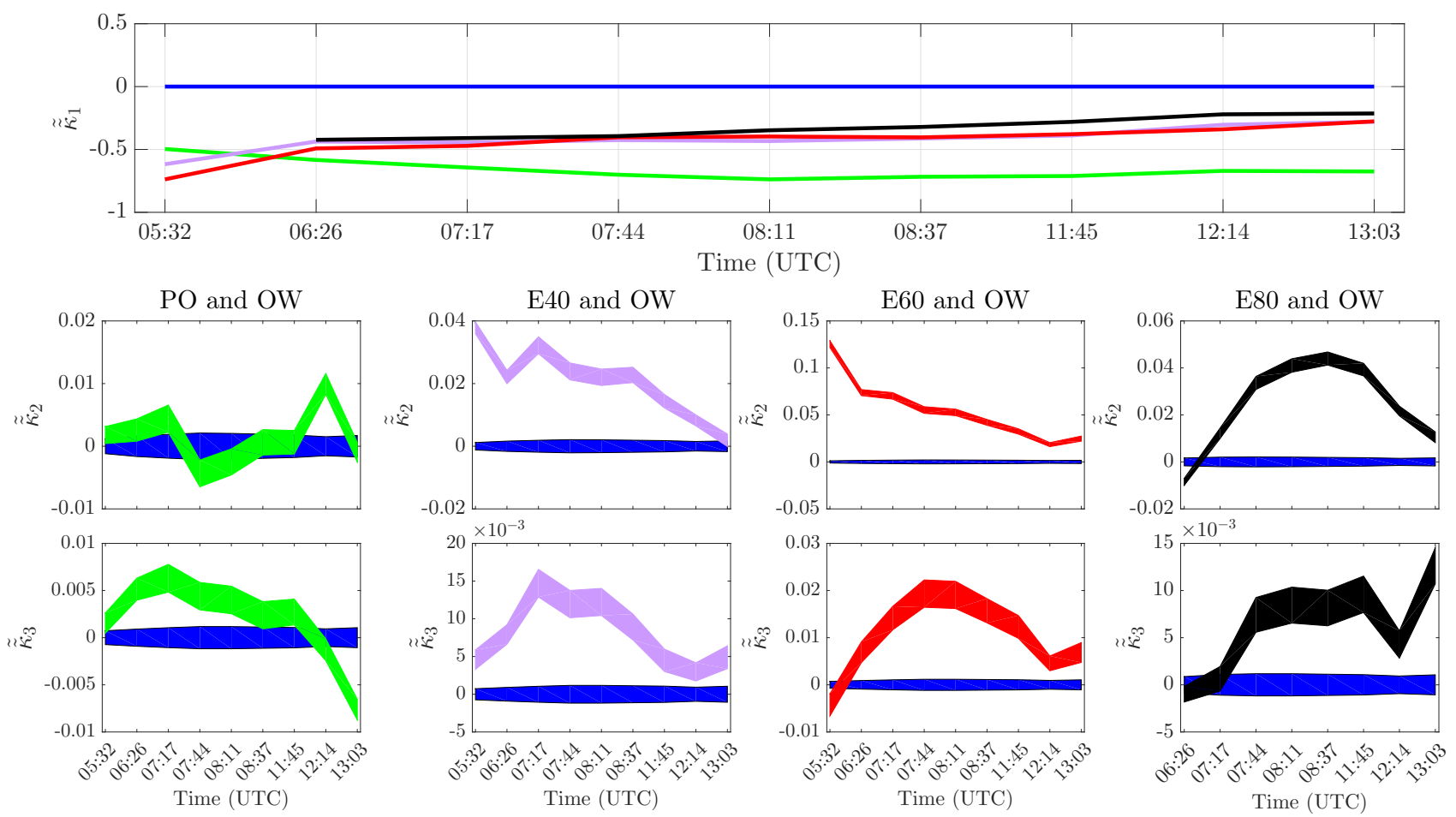

Figure 7. $\tilde{\tilde{\kappa}}_{1}, \tilde{\tilde{\kappa}}_{2}$ and $\tilde{\tilde{\kappa}}_{3}$ from the multi-looked HH intensity as a function of time. The thickness of $\tilde{\tilde{\kappa}}_{2}$ and $\tilde{\tilde{\kappa}}_{3}$ plots represents the variance within the log-cumulants. 

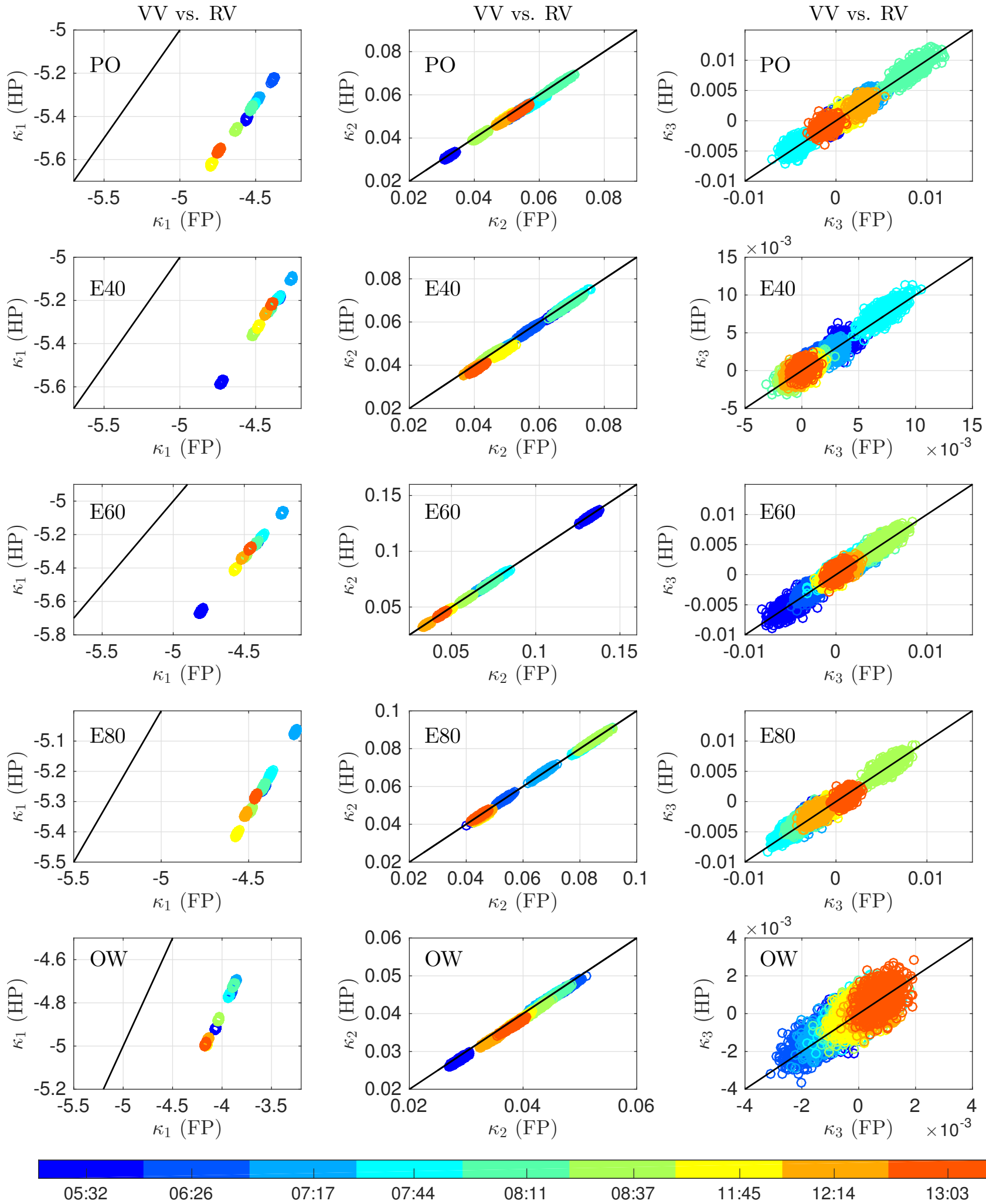

Figure 8. Scatter plots of the $\kappa_{1}, \kappa_{2}$, and $\kappa_{3}$ from the multi-looked VV and RV intensity for the different regions evaluated. 

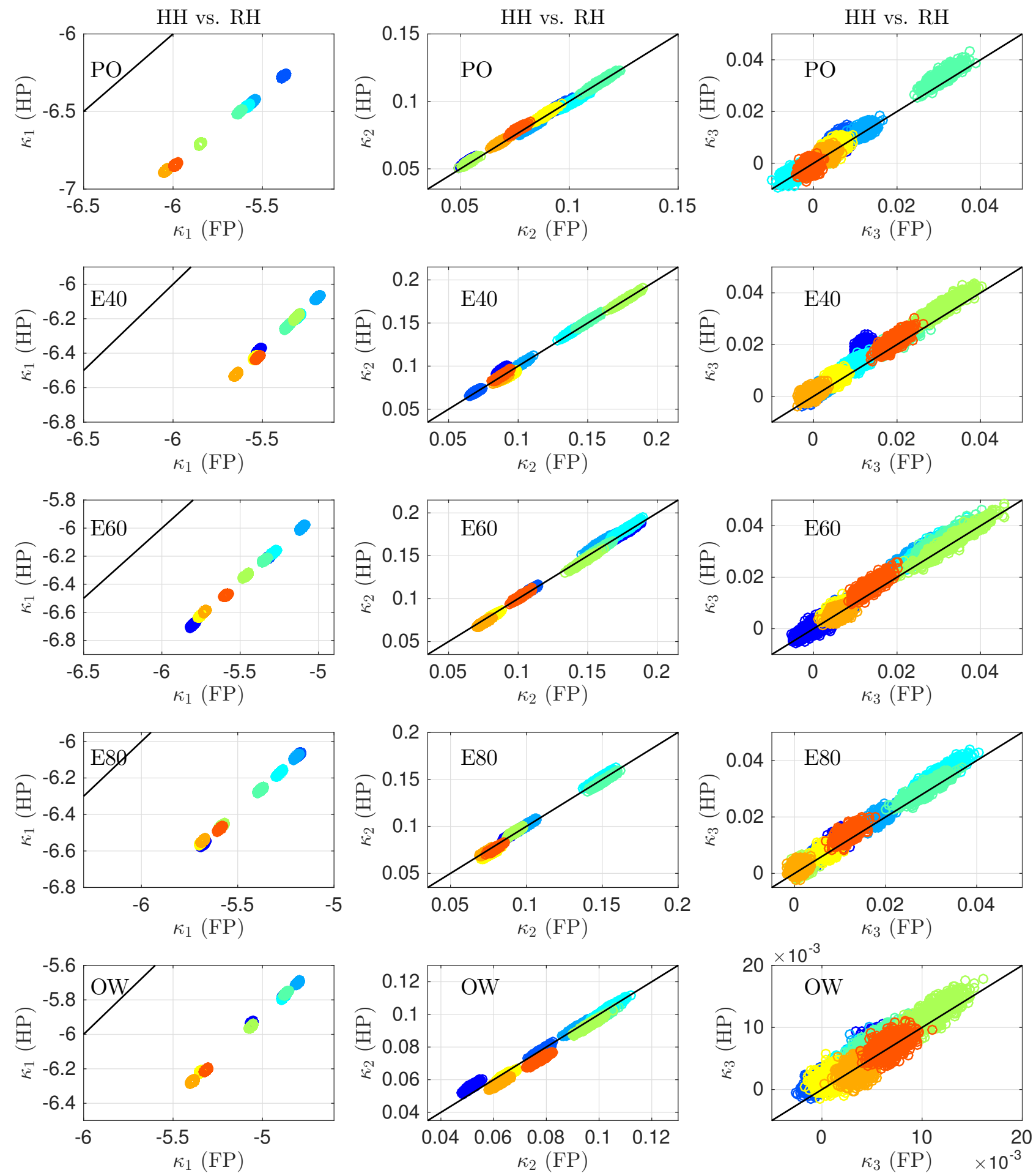

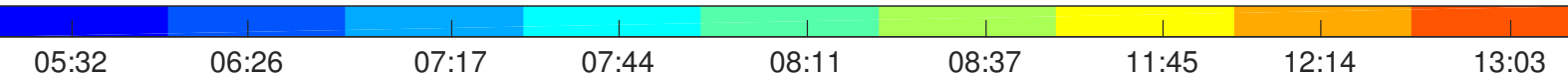

Figure 9. Scatter plots of the $\kappa_{1}, \kappa_{2}$, and $\kappa_{3}$ from the multi-looked HH and RH intensity for the different regions evaluated. 